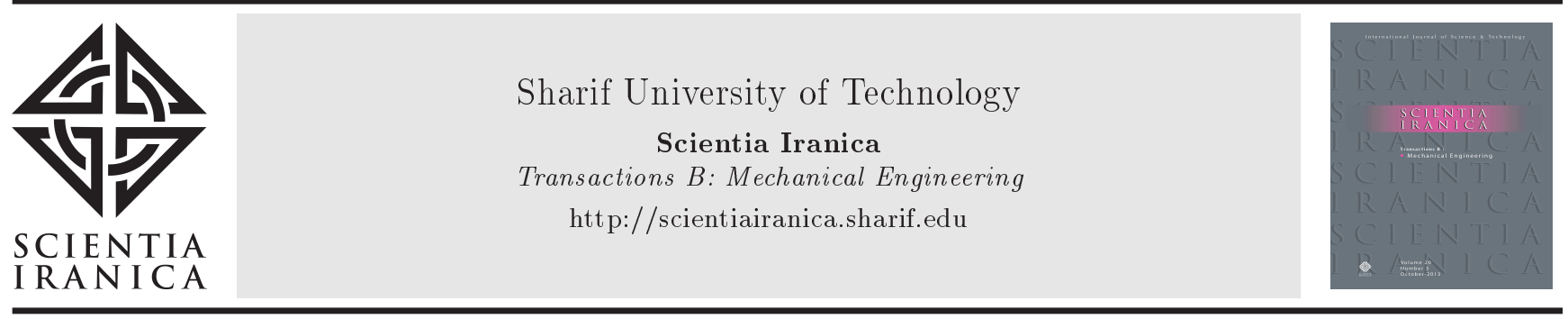

\title{
Trajectory modification of a transonic spherical projectile under hop-up mechanism
}

\author{
S.E. Salimipour, A.R. Teymourtash*, and M. Mamourian \\ Department of Mechanical Engineering, Faculty of Engineering, Ferdowsi University of Mashhad, Mashhad, P.O. Box 917ry-1111,
} Iran.

Received 19 December 2016; received in revised form 9 August 2017; accepted 5 March 2018

KEYWORDS
Projectile;
Hop-up mechanism;
Trajectory
modification;
3-D numerical
simulation;
Non-stationary
solution.

\section{Introduction}

One of the most important, challenging, and practical issues in the mechanics of flying bodies is to study the performance of projectiles. Prediction of the performance of a projectile, such as trajectory, velocity variations, altitude loss, and momentum, requires the aerodynamic and dynamic analyses by marching in space and time. Depending on their applications, projectiles have different shapes and velocities. For

\footnotetext{
*. Corresponding author. Tel.: +985138805030;

Fax: +985138763304

E-mail addresses: erfan.salimipour@gmail.com (S.E.

Salimipour); teymourtash@um.ac.ir (A.R. Teymourtash);

mamourian@um.ac.ir (M. Mamourian)
}

example, sports balls are usually spherical and have subsonic speed, and air gun pellets have smaller dimensions and transonic speed. Investigations into the in-flight motion of projectiles are conducted by researchers and many studies have been carried out in this regard in the recent years. Nietubicz and Gibeling [1] investigated the effect of thrust force on the wake area in the range of Mach numbers smaller than one to about three by numerical solution to the Navier-Stokes equations, studied the effect of fuel combustion of missile M864, and finally predicted the missile trajectory. Chakraverty et al. [2] investigated the effects of initial velocity, angle, and spin rate on the flight of a rotating spherical projectile. They computed the aerodynamic coefficients of the projectile using some empirical relations. They presented a simple mathematical treatment of the problem and its 
computer implementation using the Simulink software. Their results showed that the aerodynamic forces and moments affected the range and trajectory of the projectile flight in atmosphere. Pechier et al. [3] examined the effect of the Magnus force on the finned projectiles rotating around the longitudinal axis under the angle of attack and supersonic speeds using an unsteady algorithm based on grid movement. The most important spin-induced phenomenon was the modification of local incidences, which generated the lateral force on the tail zone. They concluded that the Magnus force generated in the finned area was more than that in the non-finned area. Silton [4] solved the Navier-Stokes equations in the range of Mach numbers smaller and larger than one at different angles of attack in order to perform aerodynamic and dynamic analyses, and they could predict the effect of longitudinal rotation of a projectile on its surrounding fluid flow. Despirito et al. [5] in a computational work showed the effect of forces and torques caused by longitudinal rotation of projectile on its dynamic stability, roll damping, Magnus, and pitch-damping moments. Their focus was primarily on the demonstration of the capability to predict the pitch-damping moment via steady-state CFD methods. $\mathrm{Yu}$ and Zhang [6] presented a numerical aerodynamic analysis of a projectile in the presence of the muzzle and calculated the quantities such as pressure, velocity, and Mach number of flow behind the projectile as well as the velocity of output stream of muzzle before exiting the projectile. The inverse Magnus effect on a rotating sphere was experimentally investigated at $\operatorname{Re}=0.6 \times 10^{5}-1.8 \times 10^{5}$ by Kim et al. [7]. The velocity field measured by Particle Image Velocimetry (PIV) indicated that the inverse Magnus effect resulted from the differences in the boundary-layer growth and separation along the retreating and advancing sphere surfaces. Pier [8] investigated the flow past a rotating sphere for $\mathrm{Re} \leq 350$ using direct numerical simulation of incompressible Navier-Stokes equations. He studied the periodic and quasi-periodic vortex shedding in the wake of the sphere and found different wake modes such as low-frequency periodic helical, quasi-periodic shedding, and high-frequency periodic helical. However, beyond $\operatorname{Re}=350$, much finer spatial meshes were required to obtain reliable results. Robinson and Robinson [9] derived the differential equations of the motion of a rotating spherical projectile in the presence of an arbitrary wind, assuming that the drag and lift forces were independent from the Reynolds number and proportional to the square of the projectile speed. They solved the equations for sports balls and predicted the trajectories. However, Jensen [10] used a dimensional analysis to show that the assumption applied in [9] was incorrect for forces dependent on the angular velocity of the projectile. Poon et al. [11] studied the flow past a rotating sphere in the Reynolds number range of 500 to 1000 and rotation rates $0 \leq \Omega \leq 1.2$ using direct numerical simulation of the Navier-Stokes equations. They succeeded in discovering a new stable regime at $\operatorname{Re}=500$ and $\Omega=1$, and at $\operatorname{Re}=640-1000$ and $\Omega \geq 0.8$. In this regime, a stable focus was observed near the onset of shear layer instability. Rafeie and Teymourtash [12] numerically examined the performance of three common samples of pellets in air gun with relatively complicated geometries in different shooting velocities at transonic regime with twodimensional simulation, and concluded that the projectile with domed nose had the best performance among all the studied samples. It is noteworthy that the above simulations were done using a set of stationary solutions, laminar flow assumption, and non-coupled solid-fluid equations. In the same year, Salimipour and Teymourtash [13] conducted a numerical simulation and compared the operations of two different sizes of the air gun pellets with calibers of 4.5 and $5.5 \mathrm{~mm}$. This simulation was carried out in a non-stationary form assuming turbulent flow and second-order time accuracy. They solved the fluid and solid equations in simultaneous coupled form in a moving computational grid and obtained more accurate results than those in [12]. Teymourtash and Salimipour [14] investigated the compressibility effects on the laminar flow past a rotating cylinder via a two-dimensional numerical simulation. The results obtained in this simulation clarified the importance of the compressibility effect on the flow and demonstrated that disregarding this effect could lead to non-physical results, which had probably been the case for the previous numerical studies. The existence of a normal shock wave could severely influence the flow field. Shrinkage of the vortex flows around the rotating cylinder due to the normal shock caused the decrease in the lift growth and, consequently, for the speed ratios higher than a critical value, the lift coefficient reached an upper bound, unlike the incompressible flows behavior.

Grid generation and flow field solution are the main issues considered in the topic of moving bodies. Mirsajedi and Hosseini [15] optimized the algorithm of an unstructured moving grid around an oscillating airfoil, so that remeshing, interpolation, and searching methods were not required in the domain. Karimian and Ardakani [16] solved two-dimensional inviscid compressible flow around a moving solid body using immersed boundary method for a Cartesian grid and prevented the accumulation of grid points using an appropriate method.

In the present study, the effect of hop-up mechanism on the performance of a spherical air gun pellet with the caliber of 4.5 millimeters is examined. Hop-up is the back-spin to increase the range of pellets via the Magnus effect, which was first used for airsoft pellets. It applies backspin to the projectile, reducing the air 
pressure on its top side. This causes the pellet to fall less over a given distance than it would without the spin applied [17]. It should be noted that the numerical simulation of the hop-up effect on the spherical air gun projectiles has not been discussed in the previous studies. Accordingly, the trajectory of an air gun projectile is improved using the hop-up mechanism. A relation is obtained between the target position and proper angular velocity generated by the hopup mechanism. Effect of the vortex shedding on the projectile motion is also analyzed. In this regard, the Navier-Stokes equations with the dynamic equations of the projectile are solved for a turbulent non-stationary compressible flow in a three-dimensional simultaneous coupled form as an FSI (Fluid-Structure Interaction) problem. In this solution, a moving coordinates system is used due to acceleration and non-stationary motion of the projectile. Aerodynamic loads resulting from numerical simulation of the Navier-Stokes equations are placed into the dynamic equations of the projectile in each time step. Then, the equations as a type of time-dependent and second-order differential equations are solved, numerically. By solving the equations, the position and the speed of the projectile are calculated at any time and, then, the values are used in the next time step for the fluid flow. This trend is continued until the projectile hits the target and, thus, time of flight, altitude loss, and hit momentum of the projectile are examined under different amounts of the hop-up. In order to validate the operation of the prepared computer code, the initial results are compared with the previous experimental data.

\section{How to create hop-up}

To create a transverse rotation perpendicular to the trajectory in spherical projectiles as pellets of air guns, a mechanism is needed in the gun barrel known as hop-up. In this mechanism, a frictional sheet may be located on the upper surface of the barrel, where it makes contact with the projectile, as shown in Figure 1; therefore, a frictional torque is created, which leads to rotation of the spherical projectile. This mechanism

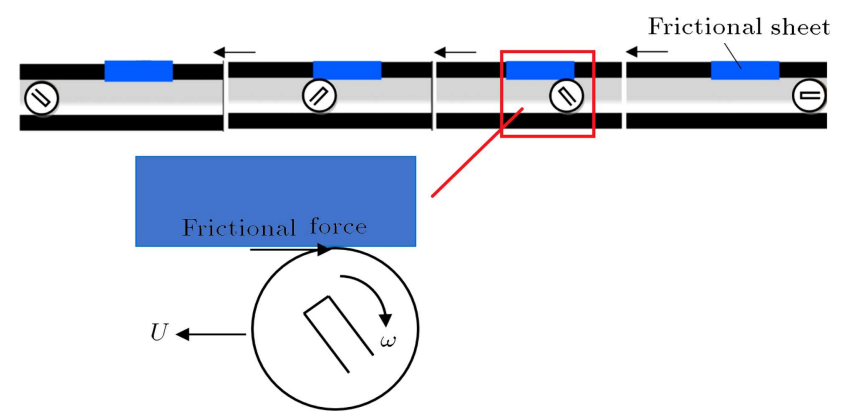

Figure 1. Projectile rotation in the barrel due to the hop-up mechanism.

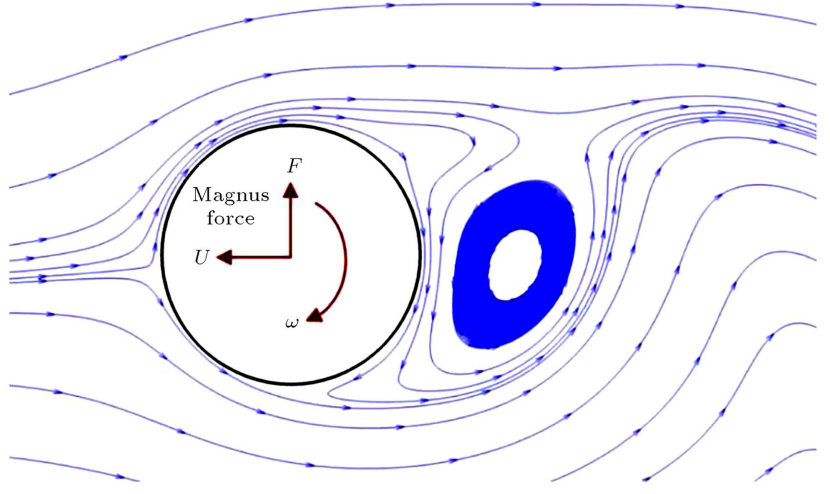

Figure 2. Magnus force generated by the hop-up mechanism.

and the generated Magnus force are shown in Figures 1 and 2, respectively. The rotational velocity of the projectile can be controlled by changing the sheet length, so that the rotational speed is increased by increasing the sheet length and lower rotational speed is applied to the projectile by decreasing the sheet length.

\section{Governing equations}

\subsection{Three-dimensional flow equations}

Integral form of the three-dimensional compressible Navier-Stokes equations in a moving coordinate system as explained in [18] is as follows:

$$
\frac{\partial}{\partial t} \int_{\Omega} \vec{W} d \Omega+\oint_{\partial \Omega}\left(\vec{F}_{c}-V_{S} \vec{W}-\vec{F}_{v}\right) d S+\int_{\Omega} \rho \vec{a}_{\Omega} d \Omega=0
$$

in which:

$$
\begin{aligned}
& \vec{W}=\left[\begin{array}{c}
\rho \\
\rho u \\
\rho v \\
\rho w \\
\rho E
\end{array}\right], \quad \vec{F}_{c}=\left[\begin{array}{c}
\rho V_{r} \\
\rho u V_{r}+n_{x} p \\
\rho v V_{r}+n_{y} p \\
\rho w V_{r}+n_{z} p \\
\rho H V_{r}+V_{S} p
\end{array}\right], \\
& \vec{F}_{v}=\left[\begin{array}{c}
0 \\
n_{x} \tau_{x x}+n_{y} \tau_{x y}+n_{z} \tau_{x z} \\
n_{x} \tau_{y x}+n_{y} \tau_{y y}+n_{z} \tau_{y z} \\
n_{x} \tau_{z x}+n_{y} \tau_{z y}+n_{z} \tau_{z z} \\
n_{x} \Theta_{x}+n_{y} \Theta_{y}+n_{z} \Theta_{z}
\end{array}\right]
\end{aligned}
$$

where $\Omega$ is the moving control volume, $\partial \Omega$ is the control surface, $V_{S}$ is the control volume velocity, $V_{r}$ is the relative velocity normal to the surface element $(d S)$, and $\vec{a}_{\Omega}$ is the acceleration of the moving control volume. The mentioned normal velocities are defined as the scalar product of the velocity vector and the unit normal vector of control surface as follows:

$$
\begin{aligned}
& V \equiv \vec{v} \cdot \vec{n}=n_{x} u+n_{y} v+n_{z} w, \\
& V_{S} \equiv \vec{v}_{p_{-} t r} \cdot \vec{n}=n_{x} u_{p_{-} t r}+n_{y} v_{p_{t} r}+n_{z} w_{p_{-} t r}, \\
& V_{r}=V-V_{S},
\end{aligned}
$$


where $\vec{v}_{p_{-} t r}$ is the translational velocity vector of the projectile. $E$ is the total energy per unit mass and is defined as:

$$
E=\frac{p}{\rho(\gamma-1)}+\left(\frac{u^{2}+v^{2}+w^{2}}{2}\right) .
$$

The shear stress components are:

$$
\begin{aligned}
& \tau_{x x}=2\left(\mu+\mu_{t}\right)\left(\frac{\partial u}{\partial x}-\frac{\vec{\nabla} \cdot \vec{v}}{3}\right) \\
& \tau_{y y}=2\left(\mu+\mu_{t}\right)\left(\frac{\partial v}{\partial y}-\frac{\vec{\nabla} \cdot \vec{v}}{3}\right) \\
& \tau_{z z}=2\left(\mu+\mu_{t}\right)\left(\frac{\partial w}{\partial z}-\frac{\vec{\nabla} \cdot \vec{v}}{3}\right) \\
& \tau_{x y}=\tau_{y x}=\left(\mu+\mu_{t}\right)\left(\frac{\partial u}{\partial y}+\frac{\partial v}{\partial x}\right) \\
& \tau_{x z}=\tau_{z x}=\left(\mu+\mu_{t}\right)\left(\frac{\partial u}{\partial z}+\frac{\partial w}{\partial x}\right) \\
& \tau_{y z}=\tau_{z y}=\left(\mu+\mu_{t}\right)\left(\frac{\partial v}{\partial z}+\frac{\partial w}{\partial y}\right)
\end{aligned}
$$

$\Theta_{x}, \Theta_{y}$, and $\Theta_{z}$ are defined as [18]:

$$
\begin{aligned}
& \Theta_{x}=u \tau_{x x}+v \tau_{x y}+w \tau_{x z}+k \frac{\partial T}{\partial x}, \\
& \Theta_{y}=u \tau_{y x}+v \tau_{y y}+w \tau_{y z}+k \frac{\partial T}{\partial y} \\
& \Theta_{z}=u \tau_{z x}+v \tau_{z y}+w \tau_{z z}+k \frac{\partial T}{\partial z} .
\end{aligned}
$$

A three-equation model for transition from laminar to turbulent flow is introduced in [19,20], which includes transport equations for the turbulent kinetic energy $\left(k_{T}\right)$, the laminar kinetic energy $\left(k_{L}\right)$, and the specific dissipation rate $(\omega)$, known as $k-k_{L}-\omega$. In this study, to simulate the transitional turbulent flow, this model is used as follows:

$$
\begin{aligned}
\frac{D\left(\rho k_{T}\right)}{D t}= & P_{k_{T}}+R_{B P}+R_{N A T}-\omega k_{T}-D_{T} \\
& +\frac{\partial}{\partial x_{j}}\left[\left(\nu+\frac{\alpha_{T}}{\sigma_{k}}\right) \frac{\partial k_{T}}{\partial x_{j}}\right], \\
\frac{D\left(\rho k_{L}\right)}{D t}= & P_{k_{L}}-R_{B P}-R_{N A T}-D_{L}+\frac{\partial}{\partial x_{j}}\left[\nu \frac{\partial k_{L}}{\partial x_{j}}\right],
\end{aligned}
$$

$$
\begin{aligned}
\frac{D(\rho \omega)}{D t}= & C_{\omega 1} \frac{\omega}{k_{T}} P_{k_{T}} \\
& +\left(\frac{C_{\omega R}}{f_{W}}-1\right) \frac{\omega}{k_{T}}\left(R_{B P}+R_{N A T}\right) \\
& -C_{\omega 2} \omega^{2}+C_{\omega 3} f_{\omega} \alpha_{T} f_{W}^{2} \frac{\sqrt{k_{T}}}{d^{3}} \\
& +\frac{\partial}{\partial x_{j}}\left[\left(\nu+\frac{\alpha_{T}}{\sigma_{\omega}}\right) \frac{\partial \omega}{\partial x_{j}}\right] .
\end{aligned}
$$

\subsection{Dynamic equations of projectile}

The velocity and acceleration relations of the projectile with respect to Figure 3 can be written as:

$$
\begin{aligned}
& \vec{v}=\dot{x} \hat{i}+\dot{y} \hat{j}+\dot{z} \hat{k}, \\
& \vec{a}=\ddot{x} \hat{i}+\ddot{y} \hat{j}+\ddot{z} \hat{k} .
\end{aligned}
$$

The forces applied by fluid to the projectile are given by:

$$
\vec{F}=F_{x} \hat{i}+F_{y} \hat{j}+F_{z} \hat{k},
$$

where $F_{x}, F_{y}$, and $F_{z}$ are the drag, lift, and lateral forces, respectively.

Based on the vectors plotted in Figure 3, it is clear that:

$$
\left\{\begin{array}{l}
m \ddot{x}=-F_{x} \\
m \ddot{y}=-m g+F_{y} \\
m \ddot{z}=F_{z} \\
I \ddot{\theta}=M_{z}
\end{array}\right.
$$

where $I$ and $M_{z}$ denote the mass inertia moment and aerodynamic torque about the $z$ axis, respectively. It should be mentioned that the aerodynamic loads $F_{x}$, $F_{y}, F_{z}$, and $M_{z}$ are functions of time and some of the flow parameters, such as velocity, pressure, and density. Therefore, differential Eqs. (21) are nonlinear.

\section{Numerical solution procedure}

The integral Eq. (1) is solved by a particular technique

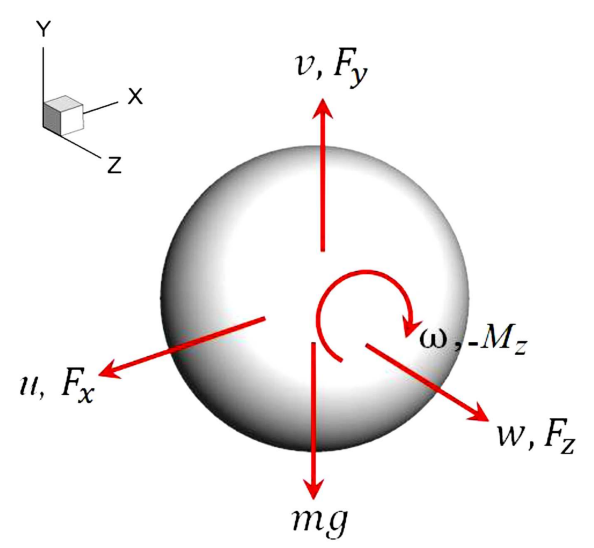

Figure 3. Diagram of the projectile motion. 


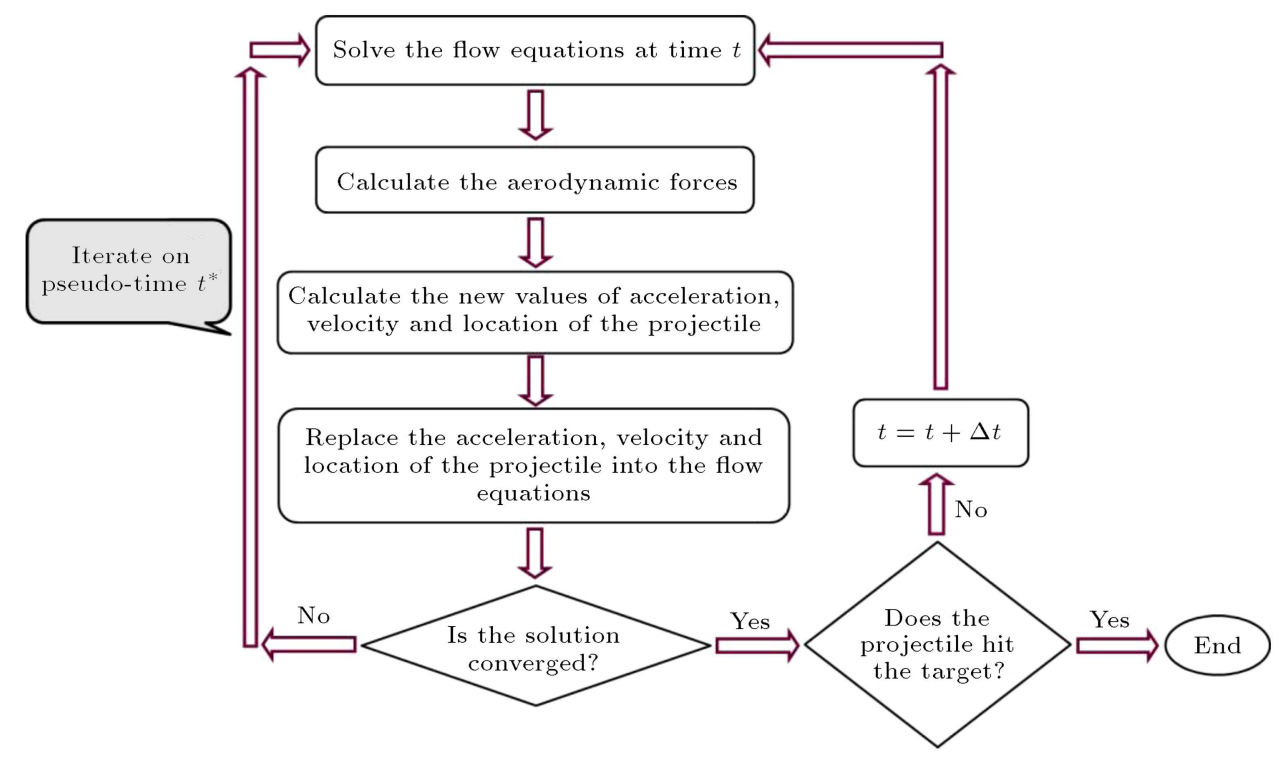

Figure 4. Simulation flowchart of the projectile motion.

known as the dual time-stepping approach, which is a common approach for considering non-stationary flows. This approach is second-order time-accurate written for an arbitrary control volume and the time level $n+1$ as [18]:

$$
\begin{aligned}
& \frac{3(\Omega M)^{n+1} \vec{W}^{n+1}-4(\Omega M)^{n} \vec{W}^{n}+(\Omega M)^{n-1} \vec{W}^{n-1}}{2 \Delta t} \\
& \quad=-\vec{R}^{n+1},
\end{aligned}
$$

where $\Delta t$ denotes the global physical time step, $M$ the mass matrix, and the superscripts $n-1, n$ and $n+1$ the time levels, respectively. The residual $\vec{R}$ is discretized using a finite-volume scheme based on the second-order Roe's approximate Riemann solver. The time-stepping approach can be written as:

$$
\frac{\partial}{\partial t^{*}}\left(\Omega^{n+1} \vec{W}^{*}\right)=-\vec{R}^{*}\left(\vec{W}^{*}\right)
$$

where $W^{*}$ is the approximation to $W^{n+1}, t^{*}$ denotes a pseudo-time variable, and $R^{*}$ is the unsteady residual, which is defined as:

$$
\vec{R}^{*}\left(\vec{W}^{*}\right)=\vec{R}\left(\vec{W}^{*}\right)+\frac{3}{2 \Delta t}(\Omega M)^{n+1} \vec{W}^{*}-\vec{Q}^{*},
$$

where:

$$
\vec{Q}^{*}=\frac{2}{\Delta t}(\Omega M)^{n} \vec{W}^{n}-\frac{1}{2 \Delta t}(\Omega M)^{n-1} \vec{W}^{n-1} .
$$

The flow equations (23), dynamic equations of the projectile (21), and the turbulence equations (15)(17) using a moving computational grid are solved simultaneously by Jameson's four-stage scheme [21].
In order to achieve the location and velocity of the projectile, the aerodynamic loads in each time step are initially obtained by solving the Navier-Stokes equations and then placed into Eqs. (21) to calculate the new values of acceleration, velocity, and location of the projectile using fourth-order Runge-Kutta method; therefore, solution preparations are provided for the next time step. The solution flowchart is shown in Figure 4. It should be noted that the velocity obtained by Eqs. (21) is applied to inner boundary of the computational grid and the far-field free-stream velocity is kept constant.

\section{Results}

\subsection{Grid generation, grid study, and boundary conditions}

For the flow solution, a spherical grid with a diameter of 30 times the projectile diameter is used, which is shown in Figure 5. According to the grid type, there is a far-field boundary and an inner boundary coinciding

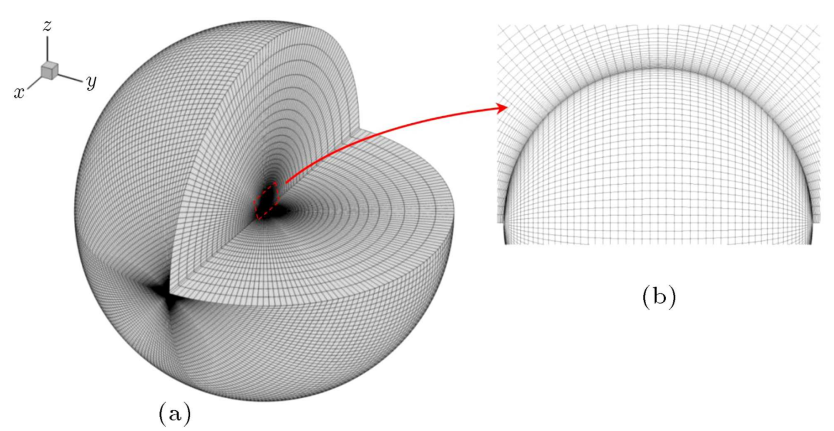

Figure 5. (a) A grid part used in flow computations. (b) A close-up view of the projectile and the grid around it. 


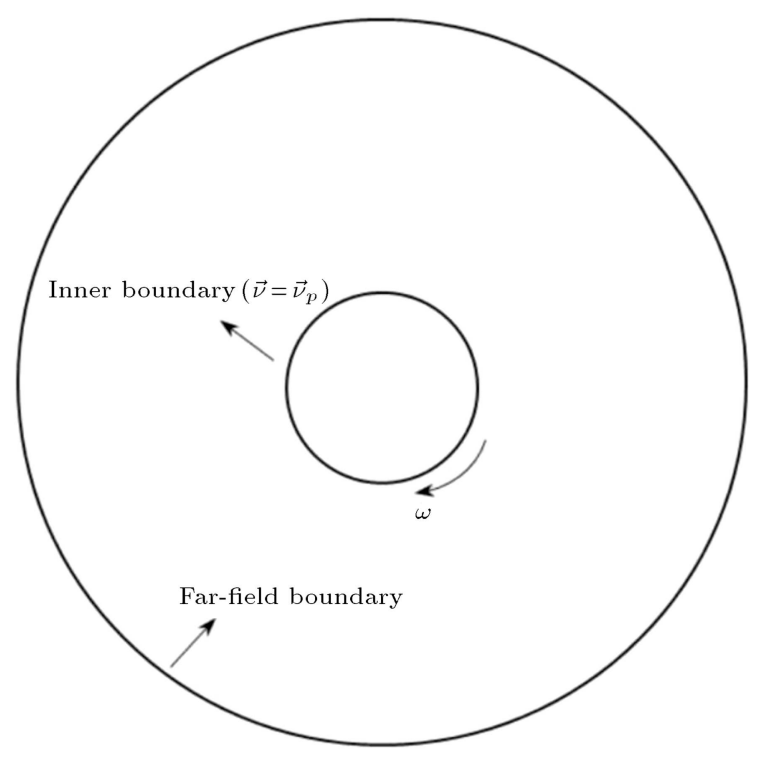

Figure 6. Boundaries of computational domain.

with the projectile surface, which is shown in Figure 6. On the outer boundary, the values of density, pressure, and velocity are as:

$$
\rho=\rho_{\infty}, \quad p=p_{\infty}, \quad T=T_{\infty}, \quad u=v=w=0 .
$$

Subscript $\infty$ implies the free-stream.

Velocity of the inner boundary $\left(\vec{v}_{i b}\right)$ is equal to the projectile velocity, including the translational and rotational motions, and normal gradients of the density, pressure, and temperature are equal to zero as follows:

$$
\begin{aligned}
& \vec{v}_{i b}=\vec{v}_{p_{-} t r}+\vec{v}_{p_{-} r o t} \\
& \left(\frac{\partial \rho}{\partial n}=\frac{\partial p}{\partial n}=\frac{\partial T}{\partial n}\right)_{i b}=0
\end{aligned}
$$

where $\vec{v}_{\text {p_rot }}$ is the rotational velocity of the sphere.

To study the computational grid independency, three grids with 400000, 720000, and 1100000 cells are examined and the results are compared with each other. Figure 7(a) and (b) compare the drag coefficient and the trajectory of a projectile with the diameter of $4.5 \mathrm{~mm}$, mass of $0.53 \mathrm{gr}$, and angular velocity of $55 \mathrm{rad} / \mathrm{s}$ in the mentioned grids under the shooting Mach number of 0.895 and shooting Reynolds of 90000 . It is observed that the results for 720000 and 1100000 cells are much close to each other. So, in this study, a grid with 720000 cells has been used.

\subsection{Code validation study}

Figure 8 shows the time variation of the drag and lift coefficients obtained by numerical solution at the shooting Mach number of 0.8 and shooting Reynolds number of 8000 , as an example. Increase in the drag coefficient after a certain time as well as oscillations of the drag and lift coefficients illustrates the vortex shedding

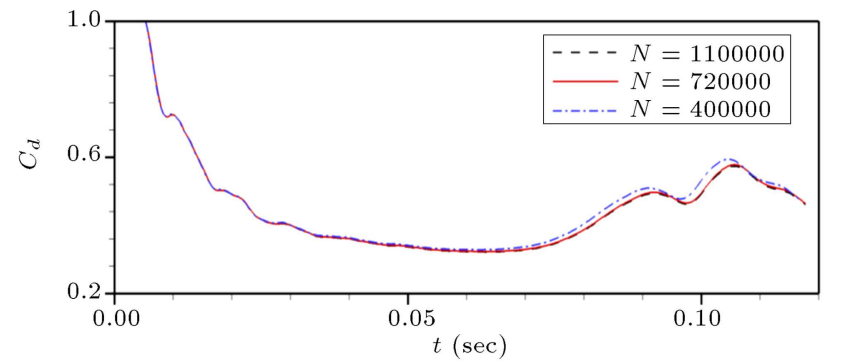

(a)

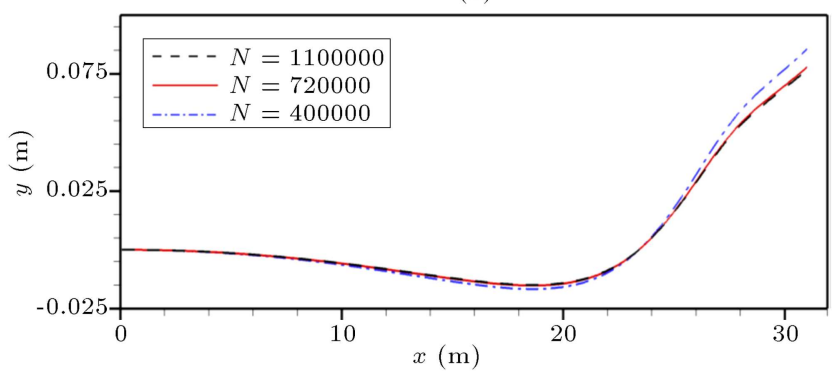

(b)

Figure 7. Comparison of (a) drag coefficients and (b) trajectories of a projectile for different computational grids with respect to time for $M_{\infty}=0.895$ and $\operatorname{Re}=90000$.

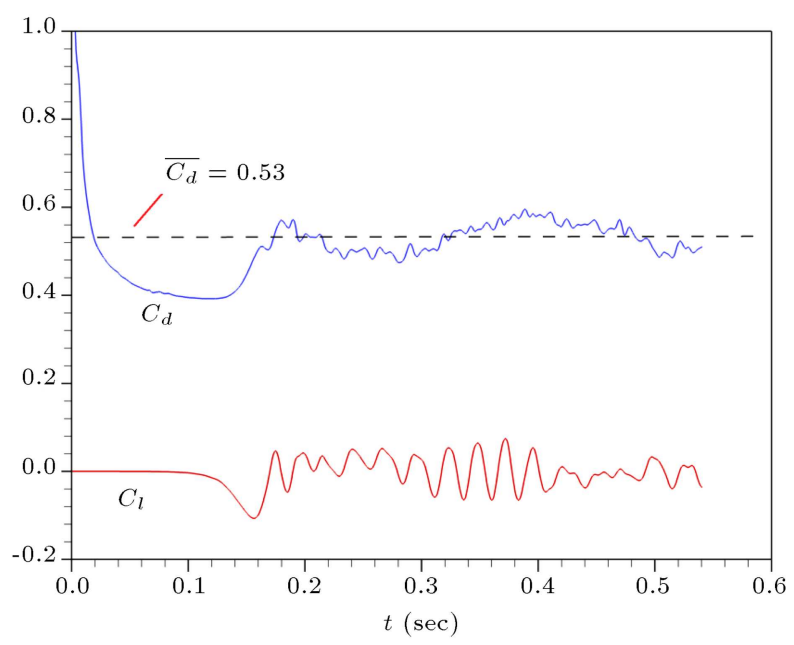

Figure 8. Temporal variations of drag and lift coefficients obtained by the present solver for $M_{\infty}=0.8$ at $\operatorname{Re}=8000$.

in the flow behind the sphere. Mean drag coefficient $\overline{C_{d}}$ is obtained by averaging the drag coefficient curve, which is shown in Figure 8. Several empirical relations are provided by Carlson and Hoglund [22], Crowe [23], Korkan et al. [24], and Henderson [25] to calculate the mean drag coefficient on a stationary sphere in the viscous compressible flow conditions. According to the comparative studies, Henderson's relation [25] has more conformity with experimental data. This relation for flows with $M_{\infty}<1$ and $100<\operatorname{Re}<10000$ is as follow:

$$
\overline{C_{d}}=24\left[\operatorname{Re}+S\left\{4.33+1.567 \exp \left(-0.247 \frac{\operatorname{Re}}{S}\right)\right\}\right]^{-1}
$$




$$
\begin{aligned}
& +\exp \left(-\frac{0.5 M_{\infty}}{\operatorname{Re}}\right) \\
& {\left[\frac{4.5+0.38(0.03 \operatorname{Re}+0.48 \sqrt{\operatorname{Re}})}{1+0.03 \operatorname{Re}+0.48 \sqrt{\operatorname{Re}}}+0.1 M_{\infty}^{2}\right.} \\
& \left.+0.2 M_{\infty}^{8}\right]+\left[1-\exp \left(-\frac{M_{\infty}}{\operatorname{Re}}\right)\right] 0.6 S,
\end{aligned}
$$

where $S=M_{\infty} \sqrt{\gamma / 2}$ is the molecular speed ratio and $\gamma$ is the ratio of specific heats.

Accordingly, the drag coefficients obtained by the numerical simulation at Reynolds number of 8000 for different Mach numbers are compared with the results achieved by Eq. (27), which are shown in Figure 9; a good agreement is obtained.

In the next step, validation of the drag and lift coefficients generated by a rotating sphere in an incompressible flow is examined. The mean drag and lift coefficients at speed ratios of $\alpha=0.2,0.3$, 0.4 , which indicate the ratio of rotational speed to translational speed of the sphere, at $\mathrm{Re}=60000$ are compared with experimental results provided by Kim et al. [7]. This experiment has been carried out on an incompressible flow and, thus, the calculations for this validation are performed at Mach number of 0.1 to prevent the influence of flow compressibility in this comparison. The comparison is presented in Table 1. It is seen that a good agreement is observed between the results by the present computer code and the experimental data.

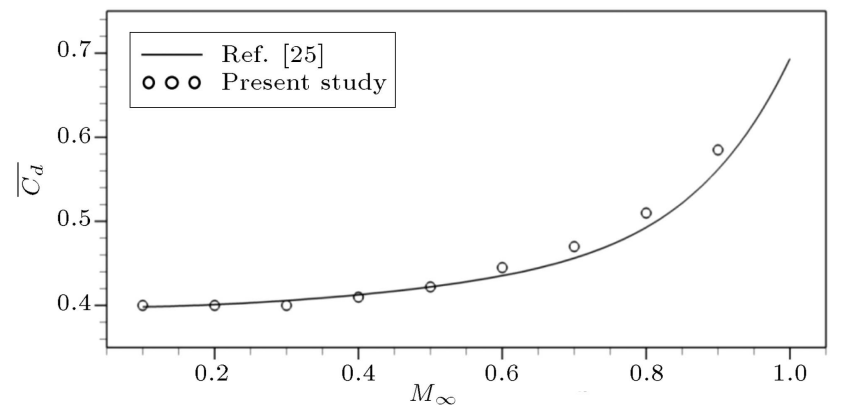

Figure 9. Comparison of mean drag coefficients obtained by the present solver and experimental data at $R e=8000$.
As seen in the above validations, accuracy of the present solver is very good. This may be due to the high resolution of the discretization method, high quality grid generation, and the use of a robust turbulence model.

\subsection{Investigation of the projectile performance}

In this section, the performance of a 0.53 gr spherical projectile with $4.5 \mathrm{~mm}$ diameter fired by an air gun without rotation and under the hop-up mechanism with various rotational speeds is investigated. Shooting speed of the projectile depends on the energy amount of the gun supplied by a capsule containing the compressed air, which is embedded in the gun body; this energy is in fact the kinetic energy of the projectile at the exit of the gun barrel, which is presented as joule or foot-pound units. The shooting speed is obtained by Eq. (28):

$$
u_{p}=\sqrt{\frac{2 \mathrm{KE}-I \omega^{2}}{m}},
$$

where KE, $m, I$, and $\omega$ denote the kinetic energy, projectile mass, mass inertia moment, and angular velocity of the projectile, respectively. In this study, assuming that the kinetic energy of the gun is 25 joules and due to the negligible value of $I \omega^{2}$, the shooting speed $\left(u_{p}\right)$ is approximately $307 \mathrm{~m} / \mathrm{s}$.

Contours of horizontal velocity component $(u)$ in two-dimensional view and instantaneous streamlines around the non-rotating sphere, drawn in Figure 10(a) and (b), indicate the pattern of the wake behind the mentioned projectile at $x=40 \mathrm{~m}$. In Figure 11, the variations of the drag and lift coefficients of the non-rotating projectile are shown at various horizontal distances $(x)$. Increase in the drag coefficient and the lift coefficient oscillation at distances greater than $30 \mathrm{~m}$ indicates the effects of the vortex shedding behind the projectile. As seen in Figure 11, the vortex shedding effects are not observed on the lift and drag coefficients for $x \leq 30$. Thus, it can be concluded that the vortex shed ding formation takes time. Mittal et al. [26] showed that by increasing the Reynolds number, the

\begin{tabular}{|c|c|c|c|c|c|c|}
\hline & \multicolumn{6}{|c|}{$\alpha$} \\
\hline & \multicolumn{2}{|c|}{0.2} & \multicolumn{2}{|c|}{0.3} & \multicolumn{2}{|c|}{0.4} \\
\hline & $\overline{C_{l}}$ & $\overline{C_{d}}$ & $\overline{C_{l}}$ & $\overline{C_{d}}$ & $\overline{C_{l}}$ & $\overline{C_{d}}$ \\
\hline Present study & 0.145 & 0.53 & 0.218 & 0.545 & 0.281 & 0.561 \\
\hline Kim et al. [7] & 0.14 & 0.525 & 0.21 & 0.54 & 0.27 & 0.555 \\
\hline Deviation (\%) & 3.5 & 0.95 & 3.8 & 0.9 & 4 & 1.08 \\
\hline
\end{tabular}
vortex shedding was begun in less time.

Table 1. Comparison of the computed mean drag and lift coefficients with experimental results for a rotating sphere at $\operatorname{Re}=60000$. 


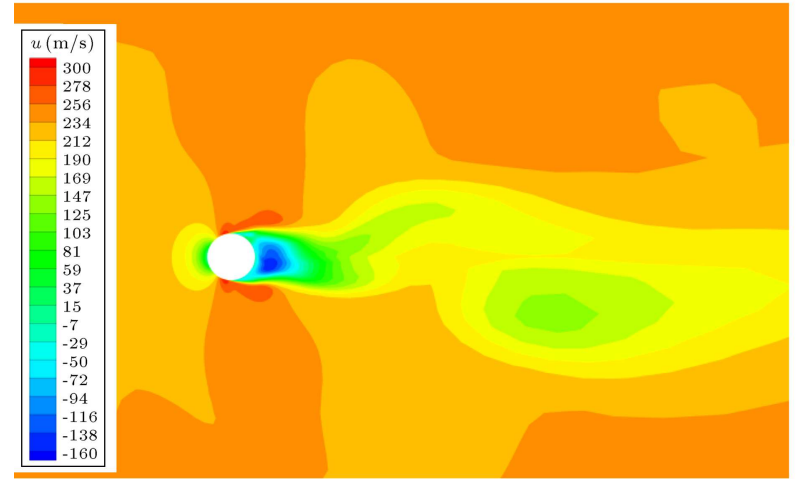

(a)

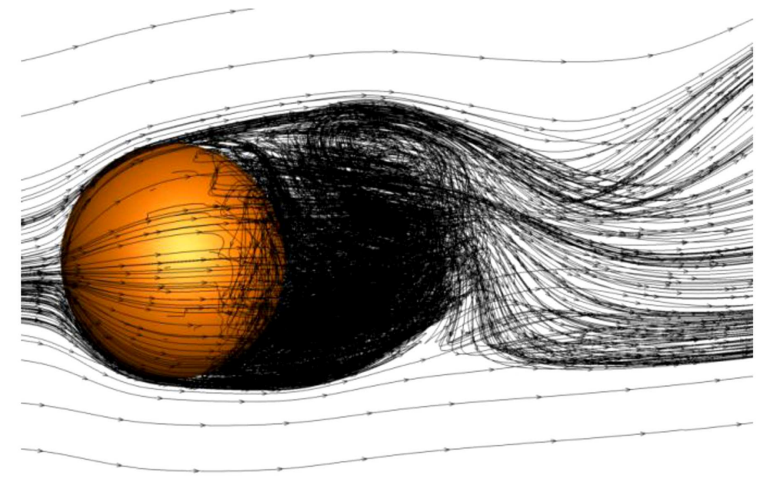

(b)

Figure 10. (a) Contours of horizontal velocity component in two-dimensional view. (b) Instantaneous streamlines around the non-rotating projectile at $x=40 \mathrm{~m}$.

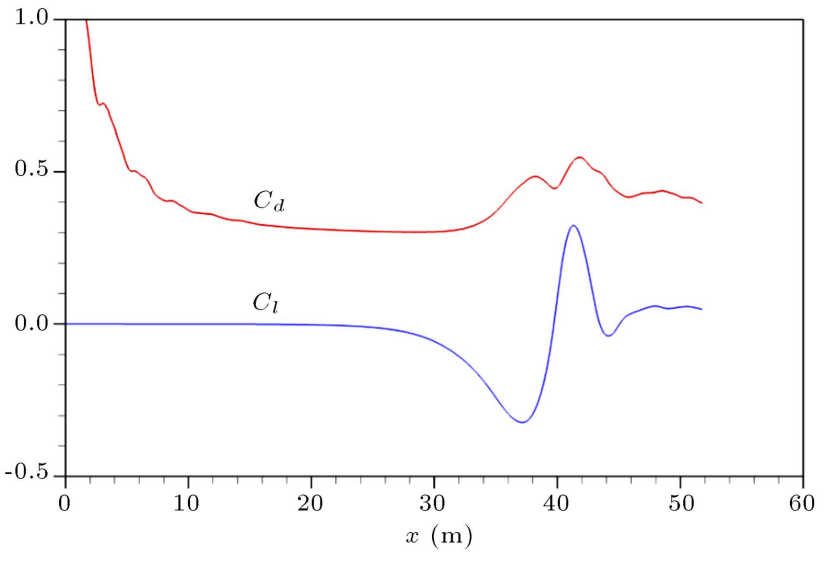

Figure 11. Variations of drag and lift coefficients of non-rotating projectile with respect to horizontal motion component.

\subsubsection{Trajectory modification of the projectile}

One of the problems in hitting the target using air guns, which is significant in competitions, is altitude loss of the pellet due to gravity. The hop-up mechanism can generate the lift force by creating a rotational speed to overcome the gravity force. Accordingly, it is necessary to adjust the hop-up applied to the projectile in a way that can compensate for the altitude loss of the projectile when hitting the target. For this purpose, different values of the rotational velocity are applied to the projectile to specify at which distance these values neutralize the altitude loss of the projectile. It should be noted that, as shown in Figure 12, the initial angular velocity applied to the projectile is nearly constant during the motion. In fact, the change of the angular velocity is negligible due to the small aerodynamic torque.

In Figure 13, the projectile trajectory is shown for different values of angular velocity in the $x-y$ plane. If the target is located on the hollow circles shown in the figure $(y=0)$, the projectile will exactly hit the target without vertical deviation. For example, if the target

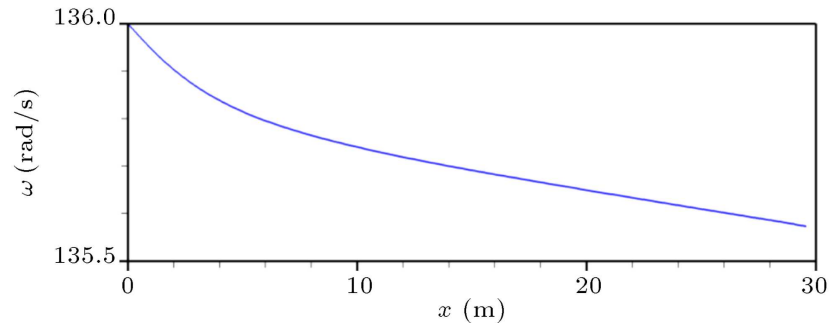

Figure 12. Variation of angular velocity at $\omega=136 \mathrm{rad} / \mathrm{s}$.

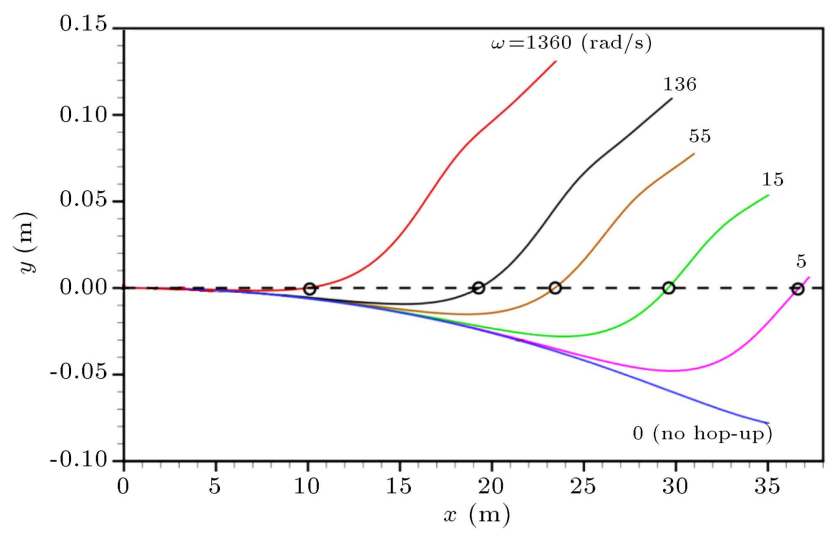

Figure 13. Projectile trajectory at various angular velocities in $x-y$ plane.

is located at $x=29.5 \mathrm{~m}$, the projectile will hit the target without altitude loss by applying proper angular velocity of $\omega=15 \mathrm{rad} / \mathrm{s}$.

As previously mentioned, the exit kinetic energy of the projectile is assumed 25 joules. To study the effect of the exit kinetic energy on the projectile performance, some results are also obtained with the exit kinetic energy of 12.5 joules. The hop-up mechanism behavior is similar to the case of 25 joules kinetic energy; however, more altitude loss is observed in the trajectory of the projectile due to less shooting speed. The result at $\omega=15 \mathrm{rad} / \mathrm{s}$ is shown in Figure 14 . 


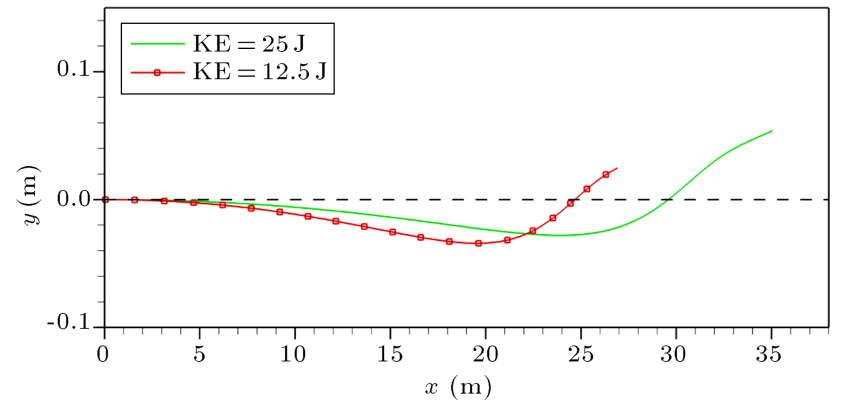

Figure 14. Projectile trajectory at two different kinetic energies at $\omega=15 \mathrm{rad} / \mathrm{s}$.

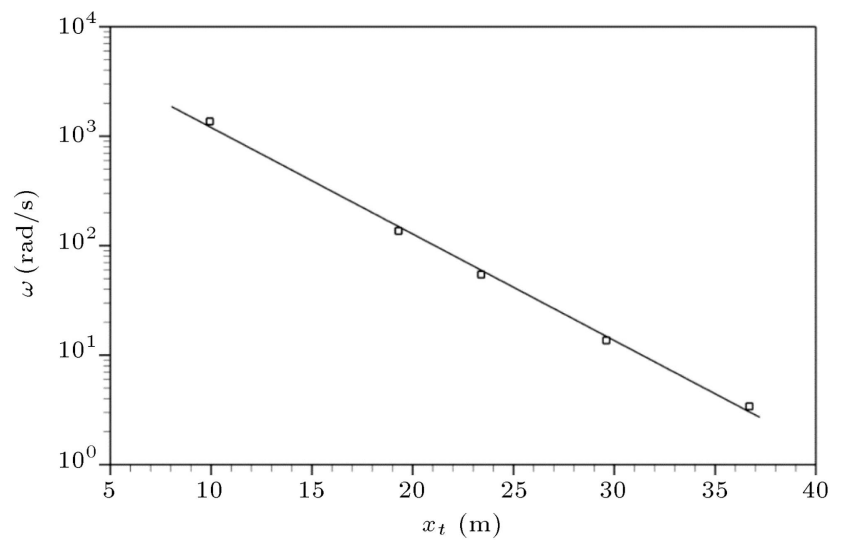

Figure 15. Relation between target position and proper angular velocities.

The proper angular velocity versus position $\left(x_{t}\right)$ is plotted in Figure 15. The squares in this figure correspond to the hollow circles drawn in Figure 12. A straight line crosses the squares with very little deviation. The equation of this line can help to find the proper angular velocity. Based on the logarithmic divisions on the vertical axis, linear equation (29) is achieved.

$$
\ln (\omega)=-0.224 x_{t}+9.33
$$

or:

$$
\omega=11285 e^{-0.224 x_{t}} .
$$

It is also observed that by moving the target away, lower angular velocity is required.

Figure 16 shows the lateral force coefficients $C_{z}$ on the projectile. These values are negligible compared to the lift and drag coefficients, which are shown in Figure 11. Therefore, they will have little effect on the horizontal motion of the projectile. The oscillatory behavior of $C_{z}$ illustrates the vortex shedding presence. It can be seen that by increasing the angular velocity, the vortex shedding onset shifts forward.

Figure 17(a) and (b) show the locations where the projectile hits the target for different values of the angular velocity at $x=20$ and $30 \mathrm{~m}$, respectively. As

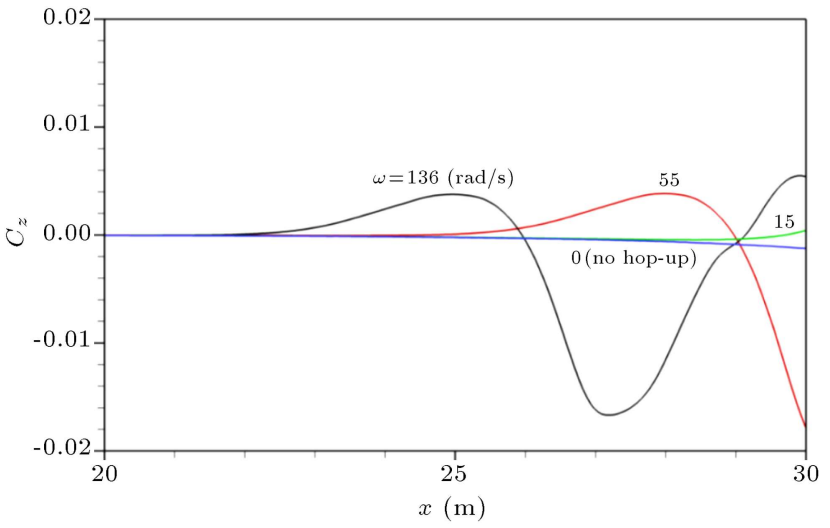

Figure 16. Lateral force coefficient with respect to horizontal motion component.

shown in these figures, there is no sensible horizontal deviation in the projectile trajectory. Figure 17(a) also demonstrates that the angular velocities less than $55 \mathrm{rad} / \mathrm{s}$ do not affect the altitude loss at $x=20 \mathrm{~m}$; but, referring to Figure 17(b), this is not applicable to $x=30 \mathrm{~m}$.

\subsubsection{Investigation of the projectile momentum}

Impact momentum of the pellet specifies its effectiveness on the target. Given the fixed projectile mass, the effectiveness is higher and the time for the projectile to hit the target is less when the hitting speed is more. Figures 18 and 19 show the variations of the Mach number and the projectile momentum at the distance of $25 \mathrm{~m}$ for various angular velocities. It is seen that the Mach number and projectile momentum are more decreased by increasing the angular velocity. The variations of the drag and lift coefficients of the projectile versus the horizontal distance are shown in Figures 20 and 21, respectively. As shown in these figures, increase in the angular velocity causes the increase in the drag coefficient; therefore, more angular velocities face larger drag coefficients and this results in more decrease in the velocity. In other words, the flight time of the projectile increases. According to Figures 20 and 21, it is observed that the variations of the drag and lift coefficients are different in various distances due to the pattern of the vortex shedding onset. For example, the difference between the drag coefficients at angular velocities of 136 and $55 \mathrm{rad} / \mathrm{s}$ at the distance of $20 \mathrm{~m}$ is more considerable than that at the distance of $23 \mathrm{~m}$. This is also true in the case of lift coefficient. Thus, the lift coefficient of the projectile with the angular velocity of $136 \mathrm{rad} / \mathrm{s}$ is lower than that with the angular velocity of $55 \mathrm{rad} / \mathrm{s}$ at the distance of $23 \mathrm{~m}$. To address this issue, contours of the pressure coefficient for the mentioned distances are compared in a two-dimensional section and shown in Figures 22 and 23. The pressure difference between the front of the projectile and behind it causes the drag, and pressure 

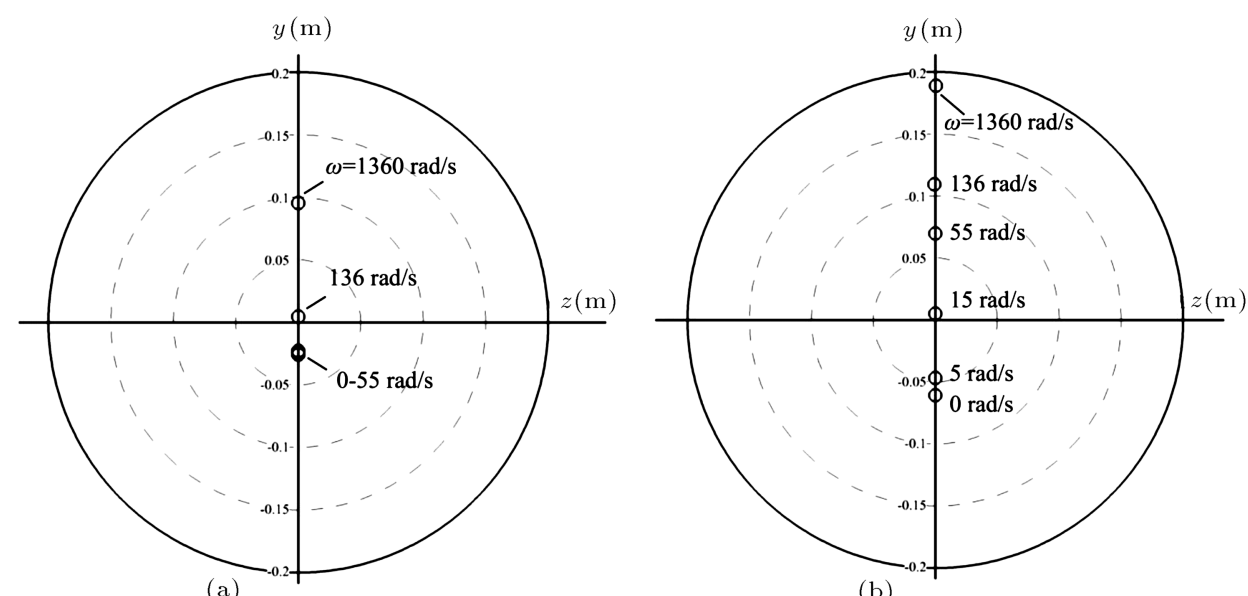

(a)

(b)

Figure 17. Locations where projectile hits the target at different angular velocities in (a) $x=20 \mathrm{~m}$ and (b) $x=30 \mathrm{~m}$.

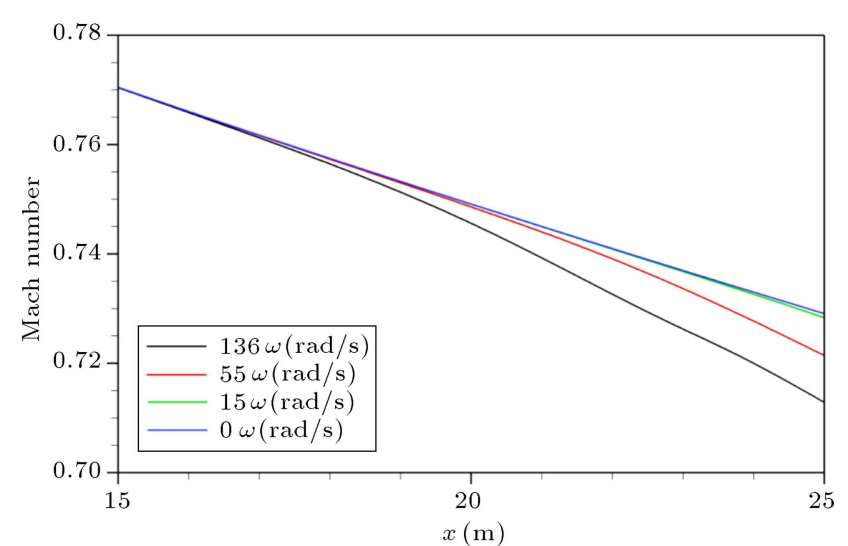

Figure 18. Projectile Mach variations at various angular velocities.

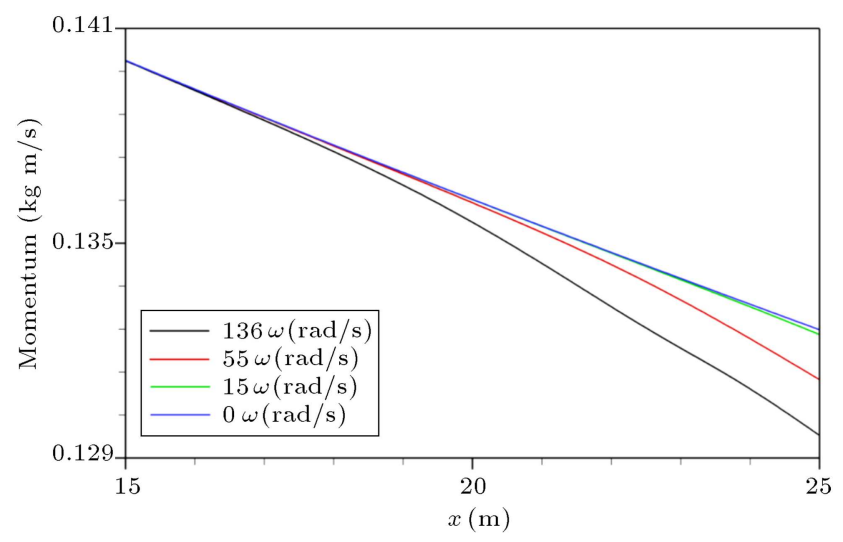

Figure 19. Projectile momentum variations at various angular velocities.

difference in upper and lower parts of the pellet brings the lift, which confirms the above explanations.

\section{Conclusion}

In this study, the performance and modifications of the trajectory of a spherical projectile used in air guns

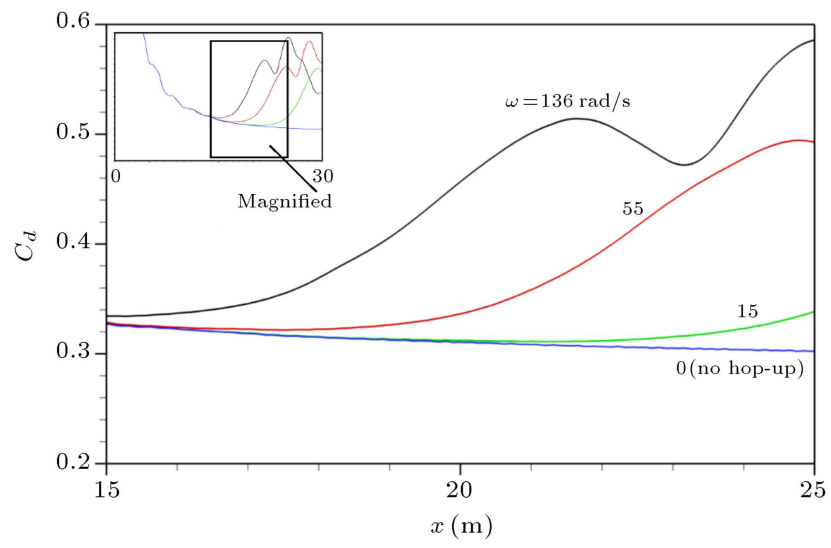

Figure 20. Drag coefficient with respect to horizontal motion component.

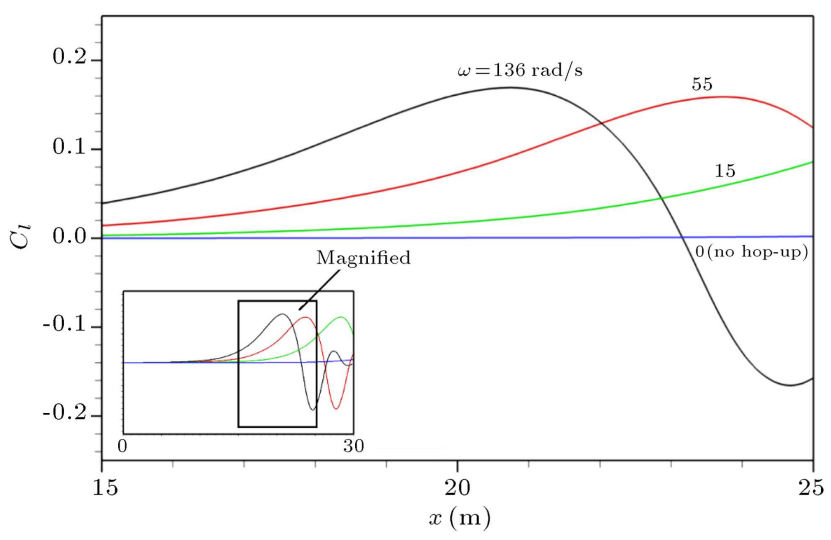

Figure 21. Lift coefficient with respect to horizontal motion component.

with the caliber of $4.5 \mathrm{~mm}$ were investigated. Primary results by the developed code were compared with experimental data and the comparisons showed a good agreement. The hop-up mechanism with several rotational velocities in transverse direction of trajectory was used to prevent the altitude loss of the projectile due to Magnus effects. variations of the drag and lift 

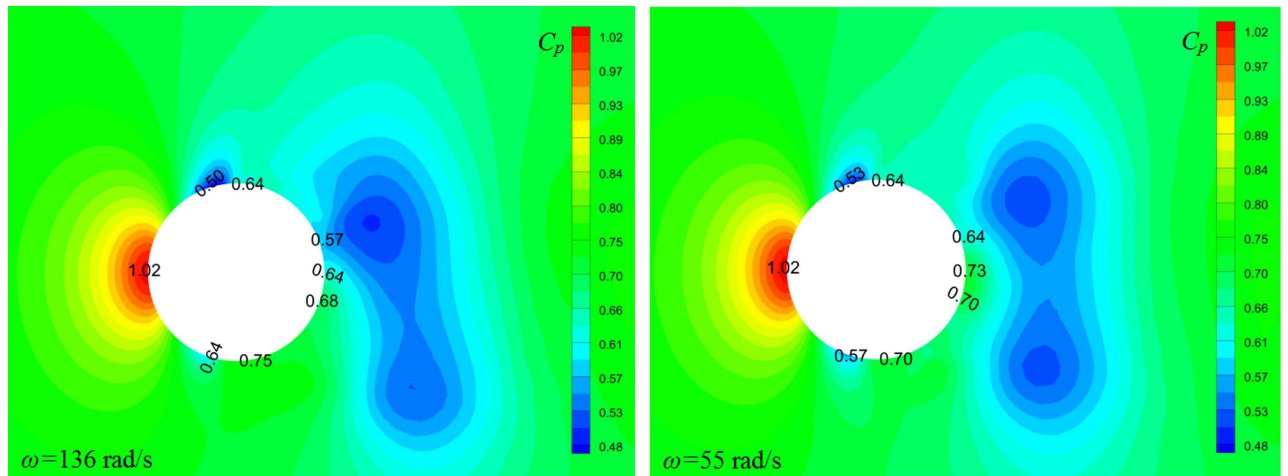

Figure 22. Contours of pressure coefficient in two-dimensional view at $x=20 \mathrm{~m}$.
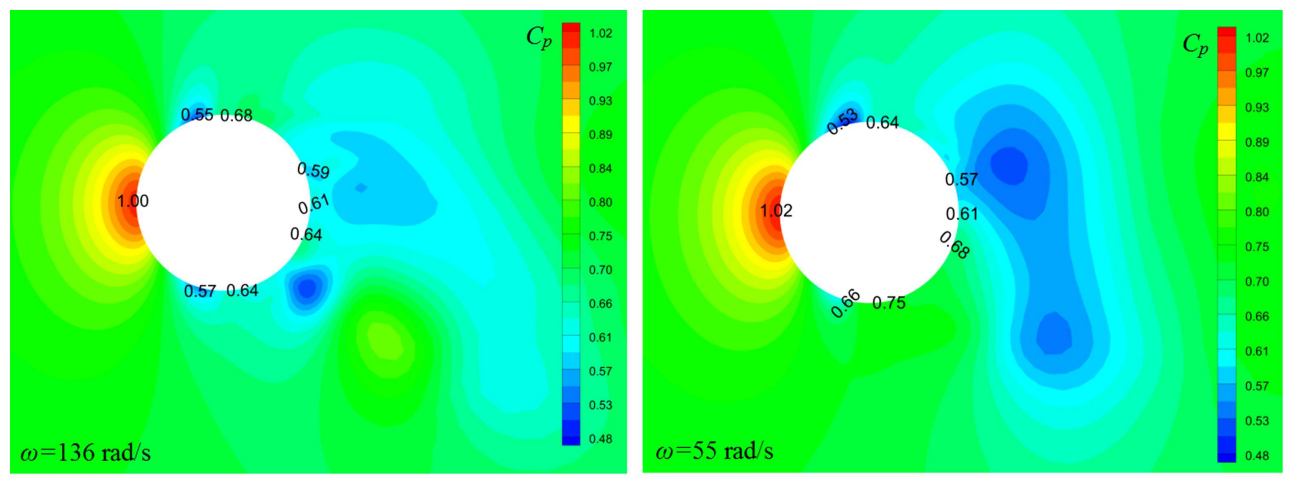

Figure 23. Contours of pressure coefficient in two-dimensional view at $x=23 \mathrm{~m}$.

coefficients were different in various distances due to the pattern of the vortex shedding onset. By increasing the angular velocity, the vortex shedding onset shifted forward and drag coefficient increased; thus, the projectile momentum decreased and the flight time of the projectile increased. In addition, a relation was obtained using data interpolation to calculate the proper angular velocity to compensate for the altitude loss of the projectile in the desired distances. It was also indicated that there was no considerable horizontal deviation in the projectile trajectory when hitting the target.

\section{References}

1. Nietubicz, C.J. and Gibeling, H.J. "Navier-Stokes computations for a reacting M864 base bleed projectile", Army Research Lab Aberdeen proving Ground MD, pp. 1-38 (1995).

2. Chakraverty, S., Stiharu, I., and Bhat, R.B. "Influence of aerodynamic loads on flight trajectory of spinning spherical projectile", AIAA Journal, 39(1), pp. 122125 (2001).

3. Pechier, M., Guillen, Ph., and Cayzac, R. "Magnus effect over finned projectiles", Journal of Spacecraft and Rockets, 38(4), pp. 542-549 (2001).

4. Silton, S.I. "Navier-Stokes computations for a spinning projectile from subsonic to supersonic speeds", Journal of Spacecraft and Rockets, 42(2), pp. 223-230 (2005).
5. DeSpirito, J., Silton, S.I., and Weinacht, P. "Navierstokes predictions of dynamic stability derivatives: evaluation of steady states methods", Journal of Spacecraft and Rockets, 46(6), pp. 1142-1154 (2009).

6. Yu, W. and Zhang, X. "Aerodynamic analysis of projectile in gun system firing process", Journal of Applied Mechanics, 77, pp. 1-8 (2010).

7. Kim, J., Park, H., Choi, H., and Yoo. J.Y. "Inverse Magnus effect on a rotating sphere", International Symposium on Turbulence and Shear Flow Phenomena, Poitiers, France (August 28-30, 2013).

8. Pier, B. "Periodic and quasiperiodic vortex shedding in the wake of a rotating sphere", Journal of Fluids and Structures, 41, pp. 43-50 (2013).

9. Robinson, G. and Robinson, I. "Comment on 'The motion of an arbitrarily rotating spherical projectile and its application to ball games", Journal of Physica Scripta, 88(1), pp. 018101-018117 (2013).

10. Jensen, J.H. "The motion of an arbitrarily rotating spherical projectile and its application to ball games", Journal of Physica Scripta, 89(6), pp. 067001-067003 (2014).

11. Poon, E.K.W., Oon, A.S.H., Giacobello, M., Iaccarino, G., and Chung, D. "Flow past a transversely rotating sphere at Reynolds numbers above the laminar regime", Journal of Fluid Mechanics, 759, pp. 751-781 (2014).

12. Rafeie, M. and Teymourtash, A.R. "The aerodynamic and dynamic analysis of three common $4.5 \mathrm{~mm}$ caliber 
pellets in a transonic flow", Scientia Iranica, Transactions B: Mechanical Engineering, 23(4), pp. 1767-1776 (2016).

13. Salimipour, S.E. and Teymourtash, A.R. "Numerical simulation and operation comparison of two sizes of air gun pellets with 4.5 and $5.5 \mathrm{~mm}$ calibers", Fluid Mech. and Aerodynamics, 3(3), pp. 35-47 (2015) (In Persian).

14. Teymourtash, A.R. and Salimipour, S.E. "Compressibility effects on the flow past a rotating cylinder", Physics of Fluids, 29, p. 016101 (2017).

15. Mirsajedi, S.M. and Hosseini Zarj, M.H. "Improvement in moving mesh algorithm around an oscillational airfoil", Aerospace Sciences and Researches, 2, pp. 7182 (2009). (In Persian)

16. Karimian, S.M.H. and Ardakani, M. "Immersed boundary method for the solution of $2 \mathrm{D}$ inviscid compressible flow using finite volume approach on moving cartesian grid", Journal of Applied Fluid Mechanics, 4(2), Special Issue, pp. 27-36 (2011).

17. Bloomfield, K., How to Win an Airsoft War: Secret Tactics for Success Revealed, Ed., 1st, Airsoftezone.com (2011).

18. Blazek, J., Computational Fluid Dynamics: Principles and Applications, Ed., 1st, Elsevier Science Ltd, pp. 212-215, 414-415, 238-241 (2001).

19. Walters, D.K. and Cokljat, D. "Three-equation eddyviscosity model for Reynolds-averaged Navier-stokes simulations of transitional flow", Journal of Fluids Engineering, 130, pp. 121401-14 (2008).

20. Furst, J. "Numerical simulation of transitional flows with laminar kinetic energy", Journal of Engineering Mechanics, 20(5), pp. 379-388 (2013).

21. Jameson, A., Schmidt, W., and Turkel, E. "Numerical solutions of the Euler equations by finite volume methods using Runge-Kutta time-stepping schemes", AIA A Paper, 81-1259 (1981).

22. Carlson, D.J. and Hoglund, R.F. "Particle drag and heat transferrin rocket nozzles", A IA A Journal, 2(11), pp. 1980-1984 (1964).

23. Crowe, C.T. "Drag coefficient of particles in a rocket nozzle", AIA A Journal, 5, pp. 1021-1022 (1967).
24. Korkan, K.D., Petrie, S.L., and Bodonyi, R.J. "Particle concentrations in high Mach number, two-phase flows", TR 74-0102, Aerospace Research Laboratories, Wright-Patterson AFB (1974).

25. Henderson, C.B. "Drag coefficients of spheres in continuum and rarefied flows", AIAA Journal, 14(6), pp. 707-708 (1976).

26. Mittal, R., Dong, H., Bozkurttas, M., Najjar, F.M., and Vargas, A. "A versatile sharp interface immersed boundary method for incompressible flows with complex boundaries", Journal of Computational Physics, 227, pp. 4825-4852 (2008).

\section{Biographies}

Seyed Erfan Salimipour received his BSc degree in Mechanical Engineering from University of Guilan, Iran, in 2005, and his MSc degree in Energy Conversion from Shiraz University, Iran, in 2009. Now, he is PhD student at Ferdowsi University of Mashhad, Iran. He has also been a lecturer in the Department of Mechanical Engineering, Quchan University of Advanced Technology, Iran, since 2009. He has already published 5 papers in international conferences and 7 journal papers.

Ali Reza Teymourtash received his BSc degree in Mechanical Engineering from Ferdowsi University of Mashhad, Iran, in 1983; his MSc degree in Mechanical Engineering (Thermo-fluids) from Sharif University of Technology, Tehran, Iran, in 1987; and his PhD degree in Energy Conversion from Ferdowsi University of Mashhad, Iran, in 2002, where he is now Associate Professor. He has published a book in Fluid Mechanics, 40 papers in respected international conference proceedings, and 30 journal papers.

Mojtaba Mamourian received his BSc degree in Mechanical Engineering in 1981, MSc degree in Mechanical Engineering in 1985, and PhD degree in Energy Conversion in 2002, all from Ferdowsi University of Mashhad, Iran, where he is now Assistant Professor. He has published 34 papers in respected international conference proceedings and 20 journal papers. 